\title{
Laboratory Profile of Women Substance Users Seeking Treatment at Tertiary Care Centre in North India: A Retrospective Study
}

\author{
Raka Jain ${ }^{1 \star}$ and Arpita Verma ${ }^{2}$ \\ ${ }^{1}$ All India Institute of Medical Sciences, Room no. 4090, $4^{\text {th }}$ Floor, \\ Teaching block, Department of Psychiatry, National Drug Dependence \\ Treatment Centre, All India Institute of Medical Sciences, New \\ Delhi-29, India \\ ${ }^{2}$ Institute for Clinical Research (India), ICRI, C-9, Central Road No. 22, \\ MIDC Industrial Area, Marol, Andheri East, Mumbai, Maharashtra \\ 400093, India
}

\section{*Corresponding author}

Raka Jain, All India Institute of Medical Sciences, Room no. 4090, $4^{\text {th }}$ Floor, Teaching block, Department of Psychiatry, National Drug Dependence Treatment Centre, All India Institute of Medical Sciences, New Delhi-29, India

Submitted: 31 Jan 2020; Accepted: 11 Feb 2020; Published: 06 Mar 2020

\begin{abstract}
Background: Women substance use is on increase worldwide. In India, published research on women substance users is limited. There is a need for quantitative and qualitative research on effective interventions for women. The present study aimed to assess the drug use pattern among women substance users based on urinalysis.
\end{abstract}

Methodology: Retrospective review of laboratory records (2007-2018) was carried out for females seeking treatment at an apex de-addiction centre of North India. Their drug use history and laboratory test results were examined. Descriptive statistics was applied using SPSS 22.0.

Results: A total of 29,339 tests were performed for drugs of abuse. Among them 415 tests (1.41\%) represented women substance users with a mean age of 33.82 (10.58) years. Female to male ratio was found to be 1:70. Majority was diagnosed for Opioid Dependence Syndrome (ODS) (56.9\%) followed by Nicotine (8.92\%), Cannabis (4.3\%), Alcohol (3.38\%), Benzodiazepines (3.13\%), and Inhalant (0.7\%) dependence. The positive urinalysis result showed $56.86 \%, 27.45 \%, 9.81 \%$, and $1.96 \%$ positive for codeine, morphine, cannabis and cotinine respectively.

Conclusion: Substance use disorders have been found to be fairly prevalent in women. Urinalysis plays a pivotal role in the clinical management of patients in substance abuse treatment programs.

\section{Introduction}

Substance use poses various kinds of problems that not only impact the individual user, but also affect the family and community. Women and men may face unique issues when it comes to substance use. Differences result from either Biology, i.e. being genetically female or male or culturally defined roles for men and women. Moreover, treatment for substance use disorders in women may progress differently than for men e.g. nicotine replacement therapies, like patches and gum, work better in men than in women [1]. For a number of reasons, among the substance abusers women comprise a unique clinical subpopulation.

The social disadvantage and subordination of women on one hand and the rapid socio-cultural and economic changes on the other, have significantly altered traditional structures and institutions within the society. Female drug users are particularly vulnerable on the route to drug dependence. Data on women substance users in India are scarce. Indian society is in transition and there are problems with changing roles and lifestyles [2]. Even then, compared to the West the problem of substance abuse among women is very low [3]. However, it is being increasingly recognized among the related professionals and the media that the prevalence of substance abuse among women is showing a definite increase [4]. According to Substance Abuse and Mental Health Services Administration (SAMHSA) 15.8 million women (or 12.9 percent) ages 18 or older have used illicit drugs in the past year. 4.6 million Women (or 3.8 percent) ages 18 and older have misused prescription drugs in the past year. Drug of abuse screening is used to screen and confirm the presence of several drugs in a patient's sample, mainly urine. Drug testing is used for clinical management of patients i.e. the patient may receive appropriate medical treatment or be screened for or monitored for illegal drug use.

Drug use among women in Asia is still considered a minor problem because the number of women classified as drug and alcohol users is estimated to be less. These figures are lower in India, but it has been suggested that this figure will increase and improved monitoring of the situation is necessary [5]. In India, studies on women substance abuse have been conducted in community settings as well as in institutional settings [6]. Most of the earlier published reports suggest that, a very small minority of women users actually seek treatment 
at substance-use treatment centres, in India.

Most of the published research work is available on pregnant women/ pre-natal care. Besides pregnancy, there is a paucity of literature among women from alcohol and drug use perspective. In addition, there is a scarcity of published work for validating substance use pattern among females. Also, most of the studies have demonstrated women substance use based on self-report, and not on urinalysis. The aim of the current study is to retrospectively examine the drug use pattern among women substance users based on urinalysis.

\section{Methodology}

The study was conducted at the National Drug Dependence and Treatment Centre (NDDTC) of the Department of Psychiatry at the All India Institute of Medical Sciences (AIIMS); a tertiarycare treatment centre of India. The services of the centre include outpatient department (OPD), inpatient, laboratory, aftercare, and liaison with other governmental and non - governmental agencies, and self-help groups. These services are provided by a team of psychiatrists, social workers, nurses, psychologists, laboratory workers and other supporting staff.

The current study included registered women patients seeking treatment between January 2007 and December 2018. For all the registered female subjects the available laboratory records pertaining to drug abuse screening were scanned and relevant information was retrieved according to a study specific pre-determined coding plan. The information included age, socio-demographic profile, substance use pattern, prescribed medicine and lab reports of Drug Abuse Screening data. Statistical analysis was performed by using Statistical Package for Social Sciences (SPSS) version 22 for Windows (Chicago, Illinois, USA).

The study was approved by the Institutional Ethics Committee. Conditions of anonymity and confidentiality were strictly adhered to during analysis and reporting of findings.

\section{Results}

A total of 29,339 urine tests were performed for screening drugs of abuse from 2007 to 2018 . Among them total number of $415(1.41 \%)$ women substance users visited the laboratory for urine tests. Female to male ratio was found to be 1:70. Mean Age of Women Substance Users $(n=415)$ was found to be $33.82(\mathrm{SD}=10.58)$ years.

Clinical profile

Number of female patients arriving for laboratory tests showed an upward trend in last five years i.e. from 2014 to 2018 . Year of 2011 reported the minimum number $(n=12)$, whereas year of 2017 reported the maximum number of women substance users $(n=55)$ (Figure I).

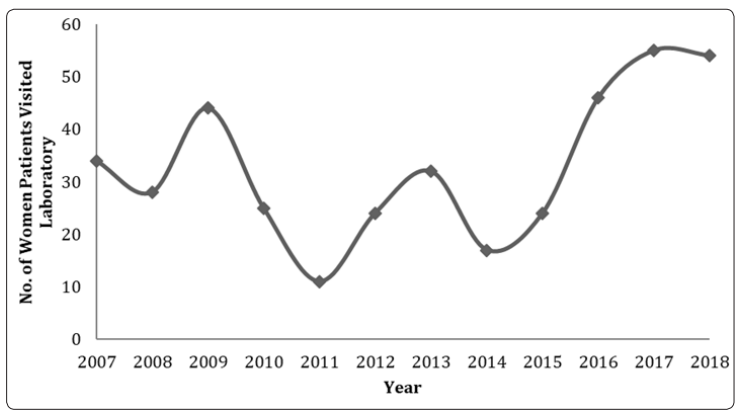

\section{Figurc}

Figure III: Tests reported positive for substance use among women patients

While testing the compliance of the prescribed medication, findings suggest that majority of tests reported positive for Benzodiazepines (32\%) followed by Propoxyphene (31\%) and Buprenorphine (17\%). Laboratory results for Pentazocine were found positive in $17 \%$ of patients. Minority of patients were reported positive for Naltrexone (2\%) and Tramadol (1\%) (Figure IV).

Figure I: Trend of female patients visiting the laboratory in 12 years 


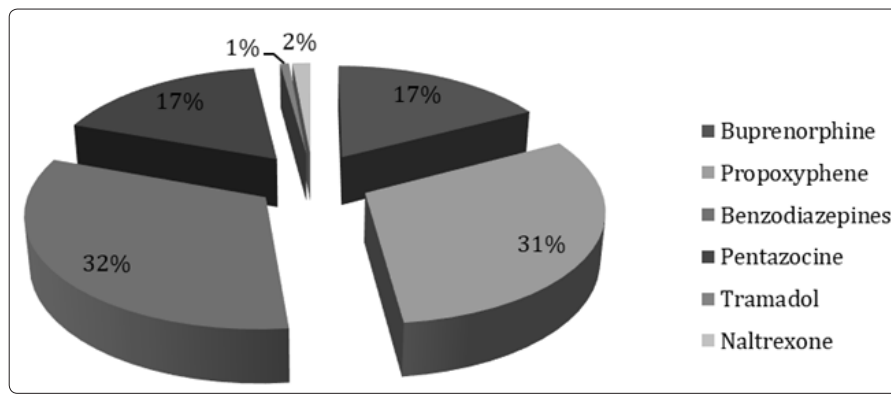

Figure IV: Tests of medicine compliance reported positive

\section{Discussion}

In many developing nations, substance use is not an exclusive or predominantly male activity anymore. However, information on substance use among women is sparse from these developing countries. Moreover, despite of many biological and social differences between men and women, for many years most animal and human research has focused mainly male subjects.

The present study was a retrospective chart review and aimed of studying the clinical and laboratory profile of women seeking help at a de-addiction setting of a tertiary care treatment centre. It attempts to provide descriptive information about substances use pattern and laboratory profile among women in northern India. This data may help to augment the available information to this hidden problem.

The main challenge in India is that a large number of people are switching over from alcohol and other illicit drugs to pharmaceutical drugs $[7,8]$. Findings suggested opioids $(56.9 \%)$ as the most common substance used which resembles to earlier Indian findings [9]. Benzodiazepines and opiates are often misused as drugs of abuse, and are not illicit, per se. The laboratory findings suggest that $32 \%$ of the patients had used benzodiazepines, $31 \%$ had used propoxyphene and $17 \%$ had used buprenorphine, are therefore a possible indication of abuse. Moreover, laboratory profile of these subjects shows $17 \%$ of women substance users were found positive for pentazocine, which may be an indicator of prescription drug misuse and in line with one of the study from the same clinical setting.

Other few Indian studies has shown the clinical data of women substance users seeking treatment at de-addiction centre and the number of subjects was much higher than the present work (12 years data) based on the laboratory findings, which indicates that the clinicians often rely on the self-report of the drug users [10]. Certain bias in self-report may not be truthful in this context. To the best of our knowledge, this is the first study of laboratory profile among women substance users.

There are certain limitations of the present study, as there was no data available on physical and psychiatric co-morbidity of the subjects. However, the use of benzodiazepines could be high because of the presence of psychiatric illness. Moreover, most of the clinical research is focused on the substance use and pregnancy among women and current work did not have any information on the pregnancy of the subjects coming to the centre.

To conclude, the society needs to accept the fact that women are vulnerable to drugs and need assistance and support in this matter. There is a still a need for quantitative and qualitative research on effective interventions for women and on how to adapt them to different countries. Current study offers insights into a rather unexplored area. The present study highlights that Urinalysis is a critical variable in substance abuse treatment programs. Laboratory plays an important role in the assessment and clinical management of patient's drug problem. Future studies with a larger sample size and more comprehensive and robust methodology is recommended $[11,12]$.

\section{References}

1. NIDA (2015) Substance Use in Women.

2. Joshi D, Rathore BS (2017) Women Substance Abuse a Rising Problem in India. International Journal of Indian Psychology 4: 166-174.

3. Kumar S, Sharma M (2008) Women and Substance Use in India and Bangladesh. Substance Use \& Misuse 43: 1062-1107.

4. Lal R, Deb KS, Kedia S (2015) Substance use in women: Current status and future directions. Indian Journal of Psychiatry 57: 275-285.

5. Nebhinani N, Sarkar S, Gupta S, Mattoo SK, Basu D (2013) Demographic and clinical profile of substance abusing women seeking treatment at a de-addiction center in north India. Indian Psychiatry Journal 22: 12-16.

6. Study of substance abuse in women. In: Women and drug abuse in India: The problem in India. Ministry of Social Justice end Empowerment, Government of India and the United Nations International Drug Control Programme, Regional Office for South Asia (UNDCP-ROSA); 2002.

7. Dayal P, Balhara YPS (2013) Profile of female patients seeking in-patient treatment for prescription opioid abuse from a tertiary care drug dependence treatment centre from India. Indian Journal of Medical Research 143;95-100.

8. Mc Hugh RK, Nielsen S, Weiss RD (2015). Prescription Drug Abuse: From Epidemiology to Public Policy. Journal of Substance Abuse and Treatment. 48: 1-7.

9. Grover S, Irpati AS, Saluja BS, Mattoo SK, Basu D (2005). Substance-dependent women attending a de-addiction center in North India: socio-demographic and clinical profile. Indian Journal of Medical Science. 59; 283-291.

10. Mandal P, Parmar A, Therthani S, Ambekar A (2016) Profile of elderly women seeking treatment for substance use disorders at a tertiary care treatment center. Journal of Substance Use 22: 353-355.

11. Ambekar A, Parmar A, Therthani S, Mandal P (2017) Profile of women substance users seeking treatment at tertiary care treatment center in India: a retrospective chart review study. Journal of Substance Use 1: 507-510.

12. Cook JL, Green CR, Ronde S, Dell CA, Graves L, et al. (2017) Screening and Management of Substance Use in Pregnancy: A Review. Journal of Obstetrics and Gynaecology Canada 39: 897-905.

Copyright: (02020 Raka Jain. This is an open-access article distributed under the terms of the Creative Commons Attribution License, which permits unrestricted use, distribution, and reproduction in any medium, provided the original author and source are credited. 